\title{
Translocation of GluR1-Containing AMPA Receptors to a Spinal Nociceptive Synapse during Acute Noxious Stimulation
}

\author{
Max Larsson $^{1,2}$ and Jonas Broman ${ }^{1,3}$ \\ ${ }^{1}$ Department of Experimental Medical Science, Division of Neuroscience, Pain Research Center, Lund University, SE-221 84 Lund, Sweden, ${ }^{2}$ Department of \\ Anatomy and Centre for Molecular Biology and Neuroscience, University of Oslo, N-0317 Oslo, Norway, and ${ }^{3}$ Department of Neuroscience, Karolinska \\ Institute, S-171 77 Stockholm, Sweden
}

Potentiation of spinal nociceptive transmission by synaptic delivery of AMPA receptors, via an NMDA receptor- and Ca ${ }^{2+} / \mathrm{calmodulin-}^{-}$ dependent protein kinase II (CaMKII)-dependent pathway, has been proposed to underlie certain forms of hyperalgesia, the enhanced pain sensitivity that may accompany inflammation or tissue injury. However, the specific synaptic populations that may be subject to such plasticity have not been identified. Using neuronal tracing and postembedding immunogold labeling, we show that a model of acute inflammatory hyperalgesia is associated with an elevated density of GluR1-containing AMPA receptors, as well as an increased synaptic ratio of GluR1 to GluR2/3 subunits, at synapses established by C-fibers that lack the neuropeptide substance P. A more subtle increase in GluR1 immunolabeling was noted at synapses formed by substance P-containing nociceptors. No changes in either GluR1 or GluR2/3 contents were observed at synapses formed by low-threshold mechanosensitive primary afferent fibers. These results contrast with our previous observations in the same pain model of increased and decreased levels of activated CaMKII at synapses formed by peptidergic and nonpeptidergic nociceptive fibers, respectively, suggesting that the observed redistribution of AMPA receptor subunits does not depend on postsynaptic CaMKII activity. The present ultrastructural evidence of topographically specific, activity-dependent insertion of GluR1-containing AMPA receptors at a central synapse suggests that potentiation of nonpeptidergic C-fiber synapses by this mechanism contributes to inflammatory pain.

Key words: central sensitization; dorsal horn; capsaicin; synaptic plasticity; LTP; electron microscopy

\section{Introduction}

Long-term plasticity of glutamatergic synapses is largely mediated by rapid regulation of the density of AMPA receptors in the postsynaptic membrane [for review, see Derkach et al. (2007) and Citri and Malenka (2008)]. Translocation to the synapse of AMPA receptors containing the GluR1 subunit has been implicated as a key event in the initial phase of long-term plasticity at some forebrain synapses, although the extent to which this mechanism operates at glutamatergic synapses in different regions of the CNS and during different stimulation paradigms is unclear.

Intense noxious stimulation that threatens tissue integrity may sensitize neuronal responses within the spinal cord dorsal horn. This central sensitization underlies some forms of hyperalgesia, in which subsequent noxious stimuli generate an enhanced sensation of pain, and is thought to involve potentiation of noci-

Received March 8, 2007; revised May 28, 2008; accepted June 2, 2008.

This work was supported by the Swedish Research Council (project number 14276), the Crafoord Foundation, the Thorsten and Elsa Segerfalk Foundation, the Magnus Bergvall Foundation, and the Greta and Johan Kock Foundations. We thank Agneta Persson for expert technical assistance.

Correspondence should be addressed to Max Larsson, Department of Anatomy and Centre for Molecular Biology and Neuroscience, University of Oslo, P.O. Box 1105 Blindern, N-0317 Oslo, Norway. E-mail: m.d.larsson@medisin.uio.no.

DOI:10.1523/JNEUROSCI.5749-07.2008

Copyright $\odot 2008$ Society for Neuroscience $\quad$ 0270-6474/08/287084-07\$15.00/0 ceptive primary afferent synapses by a mechanism similar to hippocampal NMDA receptor-dependent long-term potentiation (LTP) (Sandkühler, 2000; Ji et al., 2003). Indeed, both central sensitization and LTP of primary afferent transmission depend on NMDA receptors and $\mathrm{Ca}^{2+} /$ calmodulin-dependent protein kinase II (CaMKII) (Randić et al., 1993; Liu and Sandkühler, 1995; Fang et al., 2002; Ikeda et al., 2003, 2006; Yang et al., 2004; Pedersen et al., 2005). A link between primary afferent LTP and central sensitization is further supported by the recent demonstration that acute inflammatory pain is accompanied by LTP of C-fiber-evoked field potentials in the superficial dorsal horn (Ikeda et al., 2006).

Nociceptive primary afferent fibers are commonly divided into two major, partially overlapping populations. The peptidergic population of nociceptive $\mathrm{C}$ and $\mathrm{A} \delta$ fibers is characterized by the expression of neuropeptides such as substance P (SP) and calcitonin gene-related peptide (CGRP), whereas a parallel projection is made by $\mathrm{C}$-fibers that show little or no expression of neuropeptides (Hunt and Mantyh, 2001). Although we know that they establish synapses with different neuronal populations in the dorsal horn, the functional differences between these nociceptive pathways remain poorly understood (Hunt and Mantyh, 2001; Julius and Basbaum, 2001; Braz et al., 2005). In particular, it is unclear to what extent modulation of synapses formed 
by the two pathways contributes to different forms of hyperalgesia.

We previously observed that after injection of capsaicin in rat hindpaw skin (a common model of acute inflammatory hyperalgesia), the level of CaMKII phosphorylated at $\mathrm{Thr}^{286 / 287}$ (pCaMKII) was elevated in the postsynaptic density (PSD) of SP-positive primary afferent synapses, whereas pCaMKII levels at nonpeptidergic C-fiber synapses instead decreased from a high basal level (Larsson and Broman, 2005, 2006). Constitutively active CaMKII induces incorporation of GluR1 at hippocampal CA1 synapses (Hayashi et al., 2000). If a similar process contributes to plasticity at nociceptive synapses in capsaicin-induced hyperalgesia, it would be expected that GluR1-containing receptors accumulate at peptidergic, but not nonpeptidergic, nociceptive synapses in this pain model. To test this hypothesis, we combined neuronal tracing and postembedding immunogold labeling to investigate possible changes in AMPA receptor subunits at primary afferent synapses after cutaneous capsaicin stimulation.

\section{Materials and Methods}

Tissue preparation. Ultrathin sections for postembedding immunogold labeling were obtained from the same tissue blocks used in the previous study (Larsson and Broman, 2006), as well as from similarly processed tissue obtained from three additional animals. Briefly, seven adult male Sprague Dawley rats ( $\sim 300 \mathrm{~g})$ were anesthetized with sodium pentobarbital (50 mg/kg, i.p.), and 2\% wheat-germ agglutinin-horseradish peroxidase (WGA-HRP; Sigma) in sterile water was injected into two to three adjacent glabrous skin sites $(0.5-1.0 \mu$ l per site) in both hindpaws. Three days later, the animals were anesthetized (sodium pentobarbital; $50 \mathrm{mg} /$ kg, i.p.), and in five of the rats, 10 or $15 \mu \mathrm{l}$ of $3 \%$ capsaicin (in $15 \%$ ethanol and 7\% Tween 80 in saline) was unilaterally injected into the same skin sites as the tracer injections. Capsaicin-stimulated skin showed intense inflammatory response within a few minutes of the injection. After $20 \mathrm{~min}$, the rats were rapidly fixed by transcardial perfusion with PBS (300 mOsm, 20-30 s) and 1\% paraformaldehyde and 1.25\% glutaraldehyde in PBS ( $\sim 1 \mathrm{~L}, 30 \mathrm{~min})$. Care was taken not to inflict any additional noxious stimulation of the hindlimbs before fixation. Experimental procedures were in accordance with institutional guidelines and were approved by the Animal Care and Use Committee of Malmö-Lund. After removal of the lumbar spinal cord, parasagittal sections $(150 \mu \mathrm{m})$ of the L4 and L5 segments were cut on a Vibratome. Tracer substance was visualized by tetramethylbenzidine histochemistry, and the reaction product was stabilized with diaminobenzidine (Larsson et al., 2001). Peroxidase-labeled sections of dorsal spinal cord were embedded by freeze substitution in Lowicryl HM20 (Electron Microscopy Sciences), as described previously (Larsson et al., 2001). Serial ultrathin sections were cut and placed on single-slot Pioloform-coated nickel or gold grids (one section per grid).

Postembedding immunogold labeling. Postembedding immunogold labeling was performed as described previously (Larsson and Broman, 2006). The rabbit anti-GluR1 and anti-GluR2/3 antibodies (both from Millipore Bioscience Research Reagents), directed against the C-terminals of respective subunit, have been extensively used for postembedding immunogold studies (Matsubara et al., 1996; Petralia et al., 1999; Takumi et al., 1999) and were used here at concentrations of 4 and $2 \mu \mathrm{g} / \mathrm{ml}$, respectively. For secondary detection, goat anti-rabbit antibody conjugated to $10 \mathrm{~nm}$ colloidal gold (GE Healthcare) 1:20 or goat anti-rabbit $\mathrm{F}(\mathrm{ab})_{2}$ conjugated to $5 \mathrm{~nm}$ gold (British Biocell) 1:40 were used. In sections adjacent to the GluR1 and GluR2/3 immunogoldlabeled sections, double immunogold labeling of SP and CGRP was performed using rat anti-SP (Millipore Bioscience Research Reagents) 1:200 and rabbit anti-CGRP (Bachem AG) 1:400 or 1:800. These antibodies have been previously used by us and others in postembedding immunogold labeling procedures (Merighi et al., 1991; Larsson and Broman, 2005,2006 ) and resulted in a similar labeling pattern to that observed with other anti-SP and anti-CGRP antibodies (Zhang et al., 1993). Goat anti-rat antibody conjugated to $5 \mathrm{~nm}$ gold (British Biocell) $1: 40$ and goat anti-rabbit antibody conjugated to $15 \mathrm{~nm}$ gold (GE Healthcare) 1:20 were used for secondary detection in these sections. Sections were examined in either a JEOL or Tecnai electron microscope. In each experiment, sections from capsaicin-injected sides and controls were treated in parallel and incubated in the same drops of reagent. Five separate GluR1 immunogold labeling experiments were performed, one for each of the five capsaicin-stimulated rats. In three of those experiments, the control section was taken from contralateral dorsal horn of the same animal, and in the remaining two the control section was obtained from an animal that had not received any capsaicin injection, to assess the possibility of a contralateral effect of capsaicin stimulation. Three GluR2/3 immunogold labeling experiments were performed, one of which used tissue from a nonstimulated animal as control. No differences were observed between experiments using either control, and therefore all experiments were pooled for analysis.

Quantitative analysis of immunogold labeling. Analysis of all immunolabeled sections was performed blind with respect to experimental condition. A total of 1636 synapses were analyzed. Synapses formed by peroxidase-labeled terminals were identified by screening either an adjacent nonimmunolabeled or SP/CGRP double-labeled section or the AMPA receptor subunit-immunolabeled section at low magnification, to avoid bias from synaptic immunolabeling. Peroxidase-labeled terminals were found predominantly in inner lamina II (IIi), although some such terminals were present in outer lamina II. Peroxidase-labeled terminals in lamina I were very rare. Although this distribution of peroxidase labeling largely corresponded to that seen in the light microscope after tetramethylbenzidine processing, the sparseness of peroxidase-labeled profiles in lamina I and outer lamina II was partly attributable to the general reduction of peroxidase reaction product occurring during the stabilization step. Most peroxidase-labeled terminals contained no or very few dense-core vesicles; to yield a more homogeneous sample of synapses, terminals containing more than three dense core vesicles were discarded from the analysis (Ribeiro-da-Silva, 2004). Synapses in lamina I formed by terminals of thin-caliber primary afferent fibers containing SP and CGRP were identified by screening the adjacent SP/CGRPimmunolabeled section in the region dorsal of the peroxidase-labeled patch. The border between lamina I and outer lamina II was not well defined, and therefore lamina I was defined as the portion of the dorsal horn within $50 \mu \mathrm{m}$ of the white matter border. Thus, some sampled $\mathrm{SP}^{+} / \mathrm{CGRP}^{+}$terminals may have located in outer lamina II. Presumed low-threshold mechanosensitive (LTM) primary afferent terminals were identified by their glomerular arrangement and light axoplasm containing small, clear vesicles and numerous mitochondria (Willis and Coggeshall, 2004). Synapses formed by such terminals were sampled in lamina IIi-IV within the same rostrocaudal boundaries as the other synaptic populations.

After identification of a terminal, electron micrographs of its synapses were obtained in the GluR1 or GluR2/3 immunogold-labeled section at a magnification of $80,000 \times$ or $43,000 \times$ (depending on microscope), yielding an image scale of $\sim 1.6 \mathrm{~nm} /$ pixel. For quantitative analysis of immunolabeling, the postsynaptic plasma membrane, or cleft face of the postsynaptic density, was outlined, and locations were recorded of the center of gold particles that could be orthogonally projected onto this outline. Gold particles within $50 \mathrm{~nm}$ on the postsynaptic side, or within $25 \mathrm{~nm}$ on the cleft side, of the postsynaptic membrane were considered to be postsynaptic. Immunonegative synapses were included in the analysis, because the relatively high frequency of falsely negative synapses resulting from the inherently inefficient labeling of the postembedding labeling method would otherwise overestimate the labeling densities of sparsely labeled synaptic populations. Analysis of the subsynaptic distribution of immunogold labeling was performed essentially as described previously (Larsson and Broman, 2006).

\section{Results}

WGA-HRP was injected bilaterally into rat hindpaw skin. After allowing sufficient time for axonal transport of the tracer to the dorsal horn, capsaicin was injected unilaterally into the same skin sites. Thereby, peroxidase labeling indicating WGA-HRP trans- 
port could be used to identify terminations of nerve fibers innervating capsaicin-stimulated or nonstimulated skin. Peroxidase label was largely confined to the inner part of lamina II, indicating that WGA-HRP was preferentially transported by thin caliber primary afferent fibers that terminate in this area (Willis and Coggeshall, 2004). Indeed, in the electron microscope we observed that most terminals containing peroxidase reaction product were central terminals of type Ia glomeruli, i.e., nonpeptidergic, presumed nociceptive C-fiber endings (Ribeiro-da-Silva, 2004). Terminals of peptidergic nociceptive fibers ending in lamina I were identified by their coexpression of SP and CGRP, whereas LTM terminals in lamina IIi-IV were identified by morphological criteria. $\mathrm{SP}^{+} / \mathrm{CGRP}^{+}$and LTM terminals were selected for analysis only if they were within an area defined by the rostrocaudal extension of the peroxidase-labeled patch (Larsson and Broman, 2006). Because different primary afferent fiber populations show similar termination patterns along the rostrocaudal and mediolateral axes of the spinal cord (Willis and Coggeshall, 2004), such terminals could be assumed to originate from nerve fibers innervating the same skin area as those that gave rise to the peroxidase ${ }^{+}$terminals.

\section{GluR1 immunogold labeling}

Synapses from all examined primary afferent synaptic populations were immunogold labeled for GluR1 in tissue sections from dorsal horn ipsilateral to capsaicin injection or control dorsal horn (Fig. 1). Peroxidase ${ }^{+}$synapses contained higher linear density of postsynaptic GluR1 immunolabeling (gold particles per micrometer of postsynaptic membrane) compared with $\mathrm{SP}^{+}$/ $\mathrm{CGRP}^{+}$and LTM synapses, consistent with earlier observations of differential GluR1 expression at synapses formed by glomerular C-fiber terminals and LTM fibers (Popratiloff et al., 1996).

In dorsal horn ipsilateral to capsaicin stimulation, the linear density of postsynaptic immunolabeling was on average $94 \%$ higher at peroxidase ${ }^{+}$synapses compared with such synapses in the corresponding control dorsal horn section. At $\mathrm{SP}^{+} / \mathrm{CGRP}^{+}$ synapses and LTM synapses, no significant difference could be detected between control dorsal horn and dorsal horn ipsilateral to the capsaicin-stimulated hindpaw (Fig. 2). The same pattern was evident for all synaptic populations with respect to the total number of gold particles per synapse (supplemental Fig. 1, available at www.jneurosci.org as supplemental material).

To determine whether the observed difference in GluR1 immunolabeling ipsilateral to capsaicin stimulation was postsynaptic or could be attributed to presynaptic changes in AMPA receptor expression, we mapped the axodendritic distribution of GluR1 immunolabeling at these synapses after capsaicin stimulation and in control tissue. At peroxidase ${ }^{+}$synapses, the number of gold particles per synapse was significantly higher 0-40 nm from the postsynaptic membrane, but not at other distances, at ipsilateral compared with control synapses, indicating a selectively postsynaptic effect of capsaicin with respect to GluR1 (Fig. $3 A$ ). A similar difference between the axodendritic distributions of the linear density of gold particles was found at $0-40 \mathrm{~nm}$ from the postsynaptic membrane (supplemental Fig. 2, available at www.jneurosci.org as supplemental material). At $\mathrm{SP}^{+} / \mathrm{CGRP}^{+}$ synapses, a small but statistically significant increase in GluR1 immunolabeling was found at $0-20 \mathrm{~nm}$ from the postsynaptic membrane, although the overall capsaicin effect was not significant (Fig. 3B). At LTM synapses, the axodendritic distributions showed a selective postsynaptic localization of GluR1 immunolabeling, but no differences between dorsal horn ipsilateral to capsaicin-injected skin and control dorsal horn (Fig. 3C).
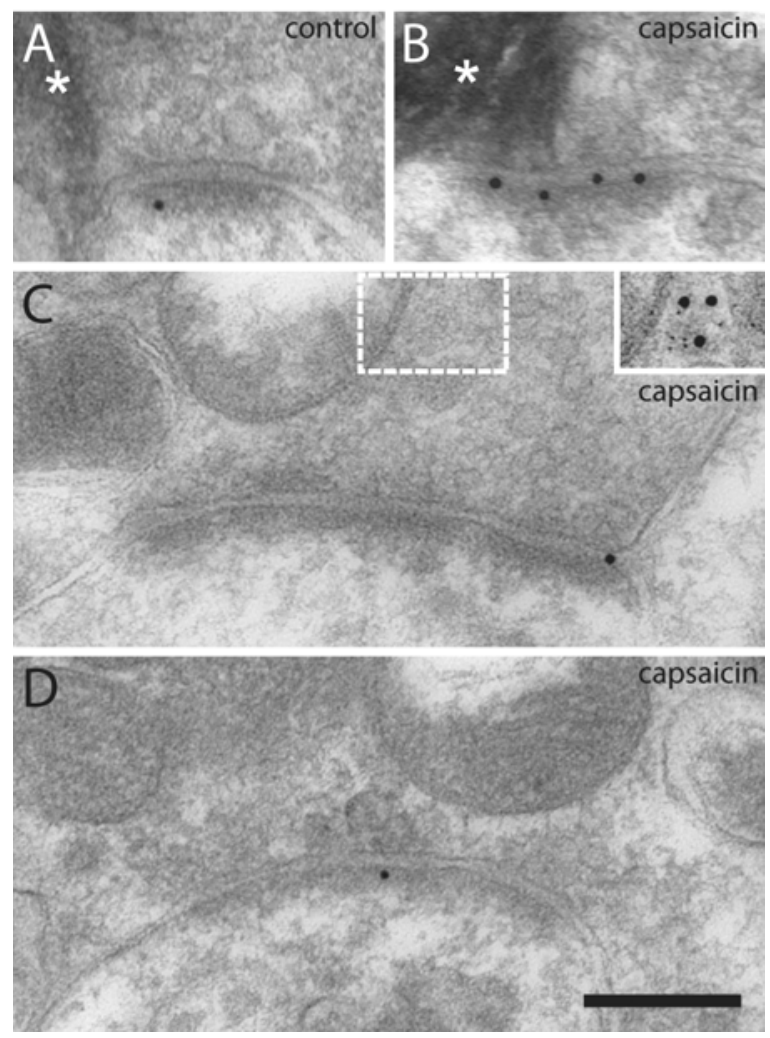

Figure 1. Examples of GluR1 postembedding immunogold labeling at identified primary afferent synapses after cutaneous capsaicin injection. $\boldsymbol{A}, \boldsymbol{B}$, Electron micrographs of immunolabeled synapses formed by peroxidase ${ }^{+}$, nonpeptidergic $C$-fiber terminals in the dorsal horn of controls $(\boldsymbol{A})$ and after capsaicin stimulation $(\boldsymbol{B})$. Asterisks indicate peroxidase reaction product. $\boldsymbol{C}$, Synapse formed by a $\mathrm{SP}^{+} / \mathrm{CGRP}^{+}$terminal in dorsal horn ipsilateral to capsaicin injection. The white frame indicates the corresponding area of the adjacent section labeled for SP ( $5 \mathrm{~nm}$ gold $)$ and CGRP ( $15 \mathrm{~nm}$ gold) that is shown in the inset. $\boldsymbol{D}$, Immunolabeled synapse formed by a presumed LTM fiber in dorsal horn ipsilateral to capsaicin-stimulated skin. Scale bar (in D): $200 \mathrm{~nm}$, valid for $\boldsymbol{A}-\boldsymbol{D}$.

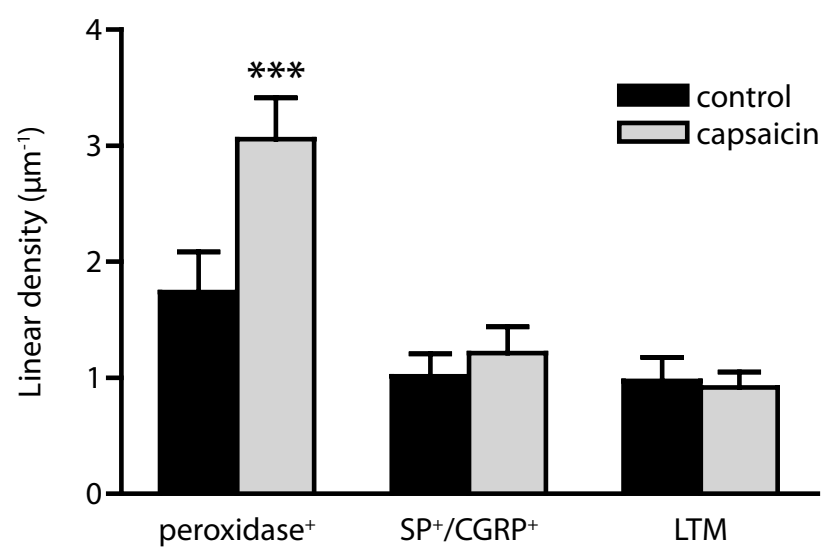

Figure 2. Quantitative analysis of primary afferent synaptic contents of GluR1 immunolabeling after capsaicin stimulation. Summed linear densities of postsynaptic GluR1 immunolabeling (gold particles per postsynaptic membrane length) in the dorsal horn of controls and after capsaicin stimulation. Error bars indicate SEM. $n=5$ individual experiments. ${ }^{* *} p<$ 0.001 , repeated-measures ANOVA followed by Bonferroni's post hoc test.

\section{GluR2/3 immunogold labeling}

To examine whether the expression of GluR2/3 subunits of the AMPA receptor was also affected at primary afferent synapses by capsaicin stimulation, we analyzed GluR2/3 immunogold labeling in ultrathin sections from three pairs of tissue blocks that had also been used for GluR1 immunolabeling (Fig. 4). In both con- 


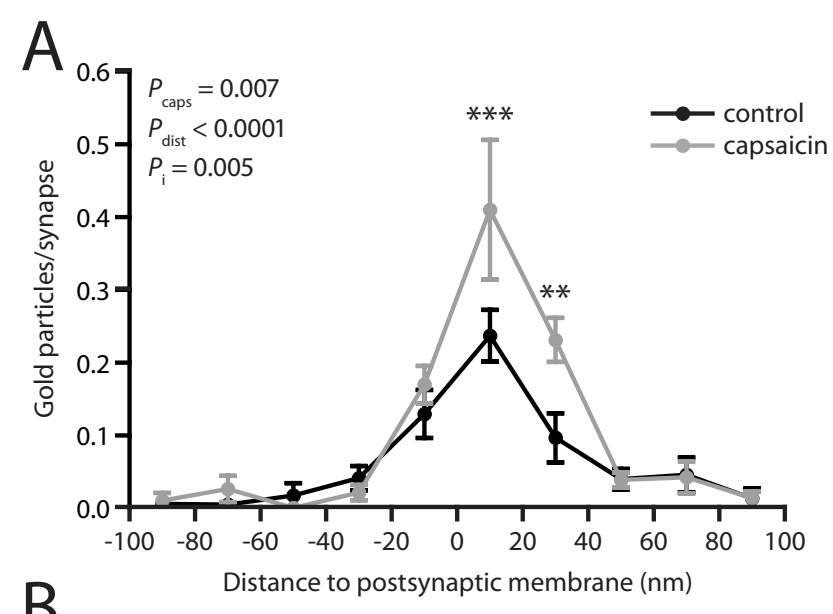

B
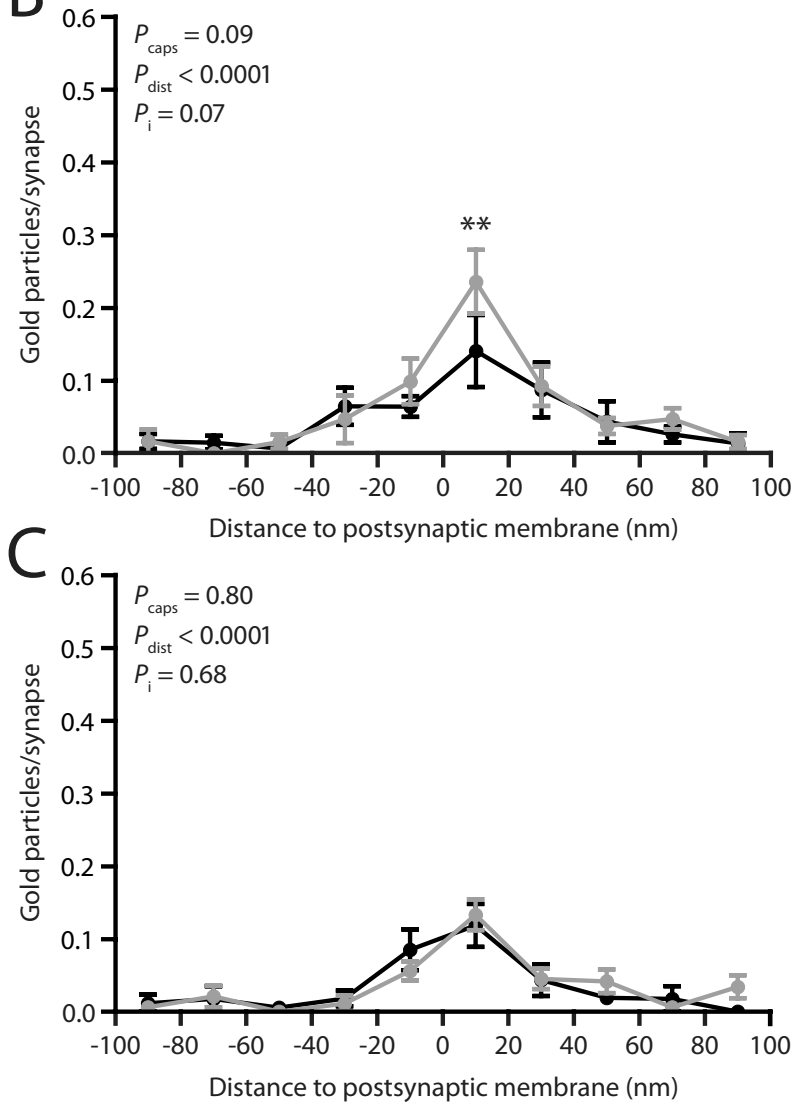

Figure 3. Axodendritic distribution of GluR1 immunolabeling at primary afferent synapses after capsaicin stimulation. $\boldsymbol{A}$, Distribution of gold particle distances to the postsynaptic membrane (presynaptic distances assigned negative value), normalized by the number of synapses, at peroxidase ${ }^{+}$ synapses. $\boldsymbol{B}$, Axodendritic distribution of GluR1 immunolabeling at $\mathrm{SP}^{+} / \mathrm{CGRP}^{+}$synapses. $\boldsymbol{C}, \mathrm{Axo} 0^{-}$ dendritic distribution of GluR1 immunolabeling at LTM synapses. Error bars indicateSEM. ${ }^{* *} p<0.01$, ${ }^{* * *} p<0.001$, two-way repeated-measures ANOVA followed by Bonferroni's post hoc test. $P_{\text {caps }}$ Statistical significance of capsaicin effect; $P_{\text {dist, }}$ statistical significance of distance effect; $P_{\mathrm{i}}$, statistical significance of interaction between capsaicin and distance effects.

trol dorsal horn and dorsal horn ipsilateral to capsaicin stimulation, $\mathrm{SP}^{+} / \mathrm{CGRP}^{+}$synapses exhibited lower linear density of gold particles signaling GluR2/3 compared with peroxidase ${ }^{+}$or LTM synapses. However, no differences in linear density (Fig. 5) or absolute number (data not shown) of gold particles between control and ipsilateral synapses could be observed at any of the examined synaptic populations.

The axodendritic distributions of GluR2/3 immunolabel-

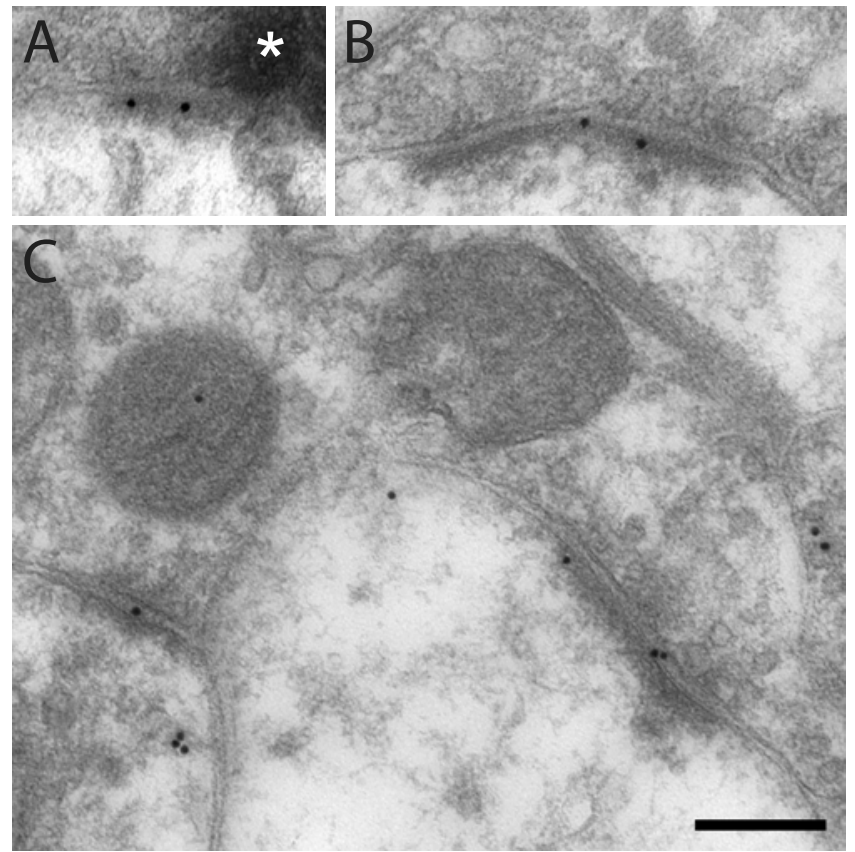

Figure 4. Examples of GluR2/3 immunolabeling at identified primary afferent synapses after cutaneous capsaicin injection. $\boldsymbol{A}$, Synapse formed by a peroxidase ${ }^{+}$nonpeptidergic $C$-fiber terminal. The asterisk indicates peroxidase reaction product. $\boldsymbol{B}$, Synapse formed by a terminal assessed to be SP ${ }^{+} / \mathrm{CGRP}^{+}$in an adjacent section (not shown). C, Immunolabeling of synapses formed by an LTM fiber. All synapses shown are from a dorsal horn section ipsilateral to capsaicin stimulation. Scale bar (in C): $200 \mathrm{~nm}$, valid for A-C.

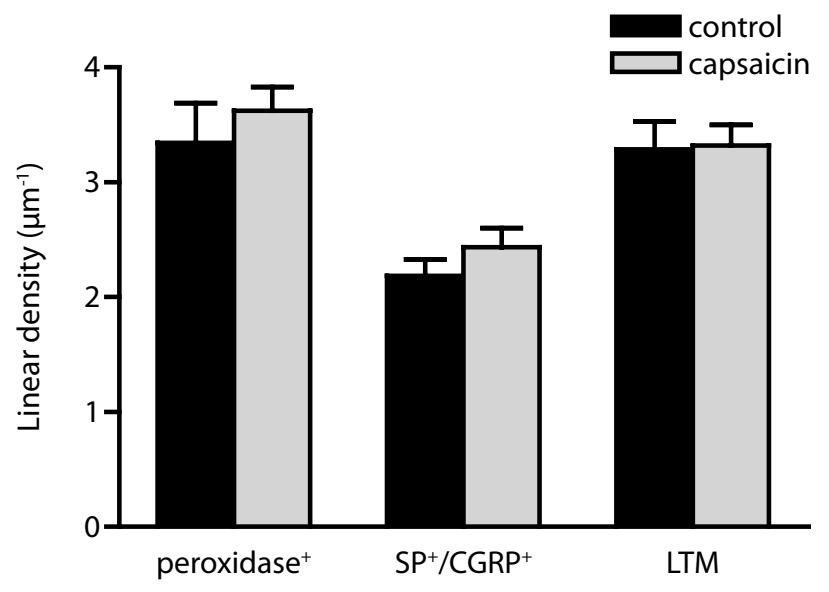

Figure 5. Quantitative analysis of primary afferent synaptic contents of GluR2/3 immunolabeling after capsaicin stimulation. Summed linear densities of postsynaptic GluR2/3 immunolabeling in the dorsal horn of controls and after capsaicin stimulation. $n=3$ individual experiments. Error bars indicate SEM. No significant differences were detected (repeated-measures ANOVA followed by Bonferroni's post hoc test).

ing were also determined (Fig. 6). Although the overall capsaicin effect on the axodendritic distribution was not significant, a slightly higher number of gold particles per synapse was observed 0-20 nm from the postsynaptic membrane at peroxidase $^{+}$synapses after capsaicin stimulation. This increase was consistent between experiments and ranged from 22 to $39 \%$. However, there was no significant difference in the linear density of immunogold labeling at any distance from the postsynaptic membrane (data not shown). Neither $\mathrm{SP}^{+} / \mathrm{CGRP}^{+}$nor LTM synapses showed any differences in axodendritic distribution of immunolabel between capsaicin-stimulated and control tissue. 

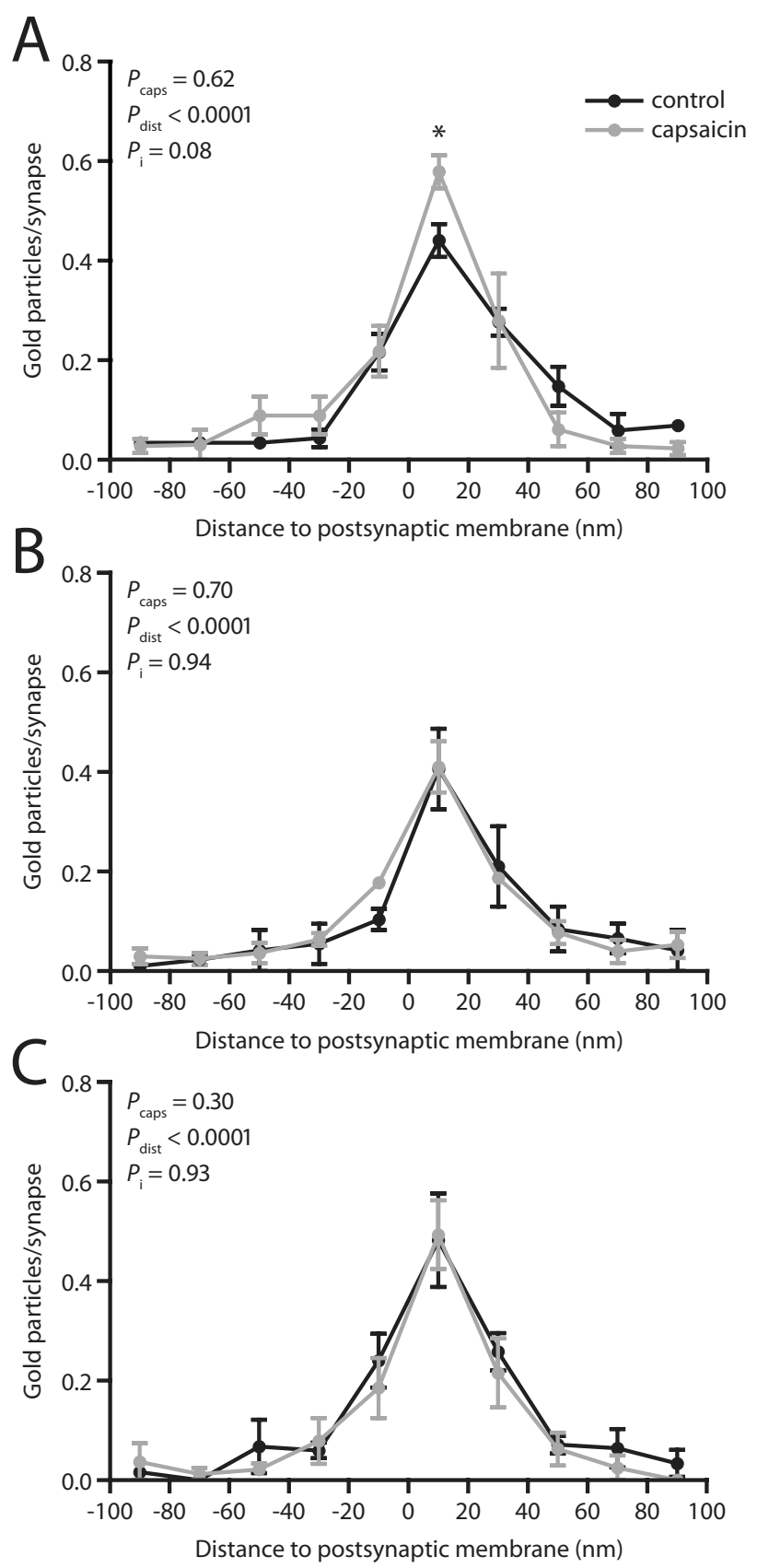

Figure 6. Axodendritic distribution of GluR2/3 immunolabeling at primary afferent synapses after capsaicin stimulation. $A$, Distribution of gold particle distances to the postsynaptic membrane (presynaptic distances assigned negative value), normalized by the number of synapses, at peroxidase ${ }^{+}$ synapses. $\boldsymbol{B}$, Axodendritic distribution of GluR2/3 immunolabeling at SP ${ }^{+} / \mathrm{CGRP}^{+}$synapses. $\boldsymbol{C}$, Axodendritic distribution of GluR2/3 immunolabeling at LTM synapses. Error bars indicate SEM. ${ }^{*} p<$ 0.05 , two-way repeated-measures ANOVA followed by Bonferroni's post hoc test. $P_{\text {caps }}$ Statistical significance of capsaicin effect; $P_{\text {dist }}$ statistical significance of distance effect; $P_{\mathrm{i}}$, statistical significance of interaction between capsaicin and distance effects.

\section{Synaptic ratio of AMPA receptor subunits}

It has been suggested by a number of studies that trafficking of different AMPA receptor subunits is differentially affected by activity, the selective synaptic delivery of GluR1-containing receptors being a prominent event in early LTP (Derkach et al., 2007; Citri and Malenka, 2008). In accordance with this notion, the clear increase in GluR1 contents observed in the present study at peroxidase $^{+}$synapses after capsaicin stimulation in contrast to the minimal increase in GluR2/3 contents at such synapses sug-

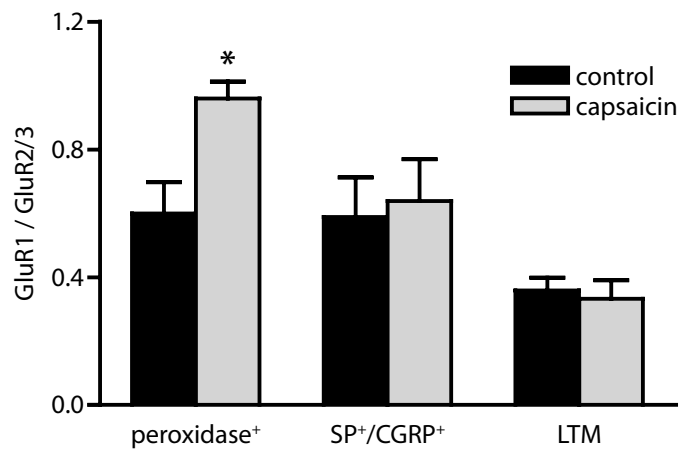

Figure 7. Altered ratio of GluR1 to GluR2/3 subunits at peroxidase ${ }^{+}$synapses after capsaicin stimulation. The ratio of GluR1 linear density (gold particles per postsynaptic membrane length) to GluR2/3 linear density was determined for each pair of stimulated and nonstimulated tissue specimens. Error bars indicate SEM. ${ }^{*} p<0.05$, repeated-measures ANOVA followed by Bonferroni's post hoc test.

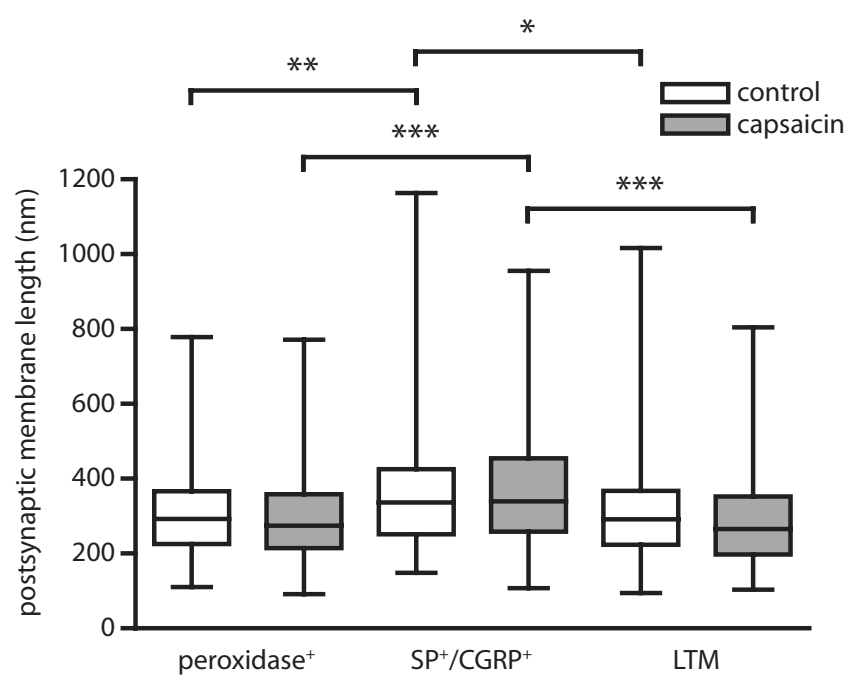

Figure 8. No change in size of primary afferent synapses after capsaicin injection. Box plot of postsynaptic membrane length as measured in the GluR1- or GluR2/3-immunolabeled section for all peroxidase ${ }^{+}, \mathrm{SP}^{+} / \mathrm{CGRP}^{+}$, and LTM synapses that had been analyzed for GluR1 or GluR2/3 immunolabel. Data from six GluR1 and GluR2/3 immunolabeling experiments were pooled. The only detected differences were those between $\mathrm{SP}^{+} / \mathrm{GRPP}^{+}$synapses and the other two synaptic populations. ${ }^{*} p<0.05,{ }^{* *} p<0.01$, ${ }^{* * *} p<0.001$, Kruskal-Wallis test followed by Dunn's post hoc test.

gested that nociceptive stimulation preferentially induced translocation of GluR1-containing receptors to this synaptic population. To examine this more directly, we determined the ratio of GluR1 to GluR2/3 linear density of immunolabeling. Indeed, an increase in the ratio of GluR1 to GluR2/3 immunolabeling was evident at peroxidase ${ }^{+}$synapses, but not at $\mathrm{SP}^{+} / \mathrm{CGRP}^{+}$or LTM synapses, after capsaicin stimulation (Fig. 7), indicating a selective translocation of GluR1 to these synapses.

\section{Synapse size}

Changes in AMPA receptor immunolabeling density in a synaptic population could be exaggerated or masked by a concomitant change in synapse size. To assess whether this could contribute to the observed changes in AMPA receptor immunoreactivity, we determined the length of postsynaptic membrane as measured in a single section through each synapse, pooling data from all GluR1 and GluR2/3 immunolabeling experiments. However, 
whereas $\mathrm{SP}^{+} / \mathrm{CGRP}^{+}$synapses were larger than peroxidase ${ }^{+}$ and LTM synapses, no changes in synapse size could be detected in any of the primary afferent synaptic populations in tissue ipsilateral to capsaicin stimulation compared with control dorsal horn (Fig. 8).

\section{Discussion}

An increasing body of evidence indicates a prominent role for trafficking of GluR1-containing AMPA receptors in NMDA receptordependent LTP and some forms of experience-dependent plasticity (Derkach et al., 2007; Citri and Malenka, 2008). Most previous studies have used immature in vitro preparations and/or different types of exogenous expression systems to assess the subunit composition of receptors translocated to synaptic populations in select forebrain regions, such as neocortex, amygdala, and the hippocampal CA1 region. The present study complements these by providing ultrastructural evidence for a relatively rapid (within 20-25 min) activitydependent delivery of native, preferentially GluR1-containing AMPA receptors to a sensory synapse in the spinal cord of adult, nontransgenic animals in vivo.

Specifically, we have shown that in the capsaicin model of acute inflammatory hyperalgesia, synapses formed by nonpeptidergic C-fibers originating from capsaicin-stimulated skin exhibit significantly higher density of GluR1, but not GluR2/3, labeling compared with such synapses in control dorsal horn. In contrast, little or no increase in GluR1 immunoreactivity was observed at peptidergic C-fiber synapses. These observations suggest a robust capsaicininduced potentiation of nonpeptidergic C-fiber synapses mediated by delivery of GluR1-containing AMPA receptors to the postsynaptic membrane. Thus, our findings support the notion that inflammatory hyperalgesia involves potentiation of transmission between nociceptive primary afferent fibers and neurons in the superficial dorsal horn (Sandkühler, 2000; Ji et al., 2003). Furthermore, phosphorylation of GluR1 at $\mathrm{Ser}^{845}$ by protein kinase A, which is necessary for synaptic incorporation of GluR1-containing AMPA receptors (Esteban et al., 2003), is induced at synaptic sites in the medial superficial dorsal horn of lumbar spinal cord after capsaicin stimulation of hindpaw skin (Nagy et al., 2004), in accordance with a translocation of AMPA receptors to somatotopically localized synapses in the superficial dorsal horn in this pain model.

Intradermal capsaicin injection induces central sensitization as well as potentiation of C-fiber evoked field potentials in the superficial spinal cord (Willis and Coggeshall, 2004; Ikeda et al., 2006). The relative contributions of different nociceptor populations to these processes are unclear. However, TRPV1, the capsaicin receptor, is expressed by large fractions of both peptidergic and nonpeptidergic nociceptive fibers in the rat (Tominaga et al., 1998; Guo et al., 1999; Michael and Priestley, 1999); furthermore, nociceptors that lack TRPV1 may be indirectly activated via the release of protons and inflammatory mediators in the peripheral tissue during inflammation (Millan, 1999; Liu et al., 2004). Thus, substantial subpopulations of both peptidergic and nonpeptidergic nociceptors were presumably activated in the present study, and differential activation of nociceptors is therefore unlikely to account for the observed differences in capsaicin-induced AMPA receptor redistribution between peptidergic and nonpeptidergic nociceptive synapses.

Previously we showed that the amounts of both total CaMKII and CaMKII phosphorylated at $\mathrm{Thr}^{286 / 287}$ (and thus autonomously active) were substantially decreased in the PSD of nonpeptidergic C-fiber synapses after capsaicin stimulation (Larsson and Broman, 2006). This apparent discrepancy might be explained by a CaMKIIindependent translocation of AMPA receptors to these synapses. For example, GluR4-containing receptors can be driven to synapses by a protein kinase A-dependent mechanism that does not involve CaMKII activation (Zhu et al., 2000; Esteban et al., 2003), indicating the possibility of CaMKII-independent translocation of AMPA receptors cocontaining GluR1 and GluR4. However, GluR4 is present at relatively low levels in the superficial dorsal horn (Tachibana et al., 1994; Nagy et al., 2004; Polgár et al., 2008), and trafficking of GluR1 to the plasma membrane of dorsal horn neurons after visceral capsaicin stimulation is sensitive to CaMKII inhibition (Galan et al., 2004), as is the central sensitization that develops after cutaneous capsaicin stimulation (Fang et al., 2002). Alternatively, AMPA receptors could be driven to $\mathrm{C}$-fiber synapses as a result of a transient increase in CaMKII autophosphorylation that subsequently falls to basal (and lower) levels within 20-25 min. Furthermore, CaMKII activated by binding $\mathrm{Ca}^{2+} /$ calmodulin or the NR2B subunit of NMDA receptors (Lisman et al., 2002) could mediate synaptic incorporation of GluR1 even in the absence of autophosphorylation of the enzyme; interestingly, autophosphorylation of the $\alpha$ isoform of CaMKII has been shown to be dispensable for several more slowly developing forms of hyperalgesia (Zeitz et al., 2004). Finally, it is possible that GluR1 delivery to these synapses requires autophosphorylation of a CaMKII pool that is located at a distance from the PSD (which our previous analysis was not designed to detect).

That we did not observe substantially changed levels of AMPA receptor subunits at $\mathrm{SP}^{+} / \mathrm{CGRP}^{+}$synapses after capsaicin stimulation is surprising, especially because lamina I projection neurons that receive preferential, potentiatable primary afferent input from $\mathrm{SP}^{+} / \mathrm{CGRP}^{+}$fibers (McLeod et al., 1998; Todd et al., 2002; Ikeda et al., 2003, 2006) are essential for the full expression of hyperalgesia (Mantyh et al., 1997; Nichols et al., 1999; Vierck et al., 2003). In addition, levels of autophosphorylated, presumably active CaMKII are increased at $\mathrm{SP}^{+} / \mathrm{CGRP}^{+}$synapses after peripheral capsaicin stimulation (Larsson and Broman, 2006), confirming that these synapses are activated in this model of inflammatory pain. The apparent failure of capsaicin stimulation to induce GluR1 accumulation at $\mathrm{SP}^{+} / \mathrm{CGRP}^{+}$synapses could be attributable to a slower mode of translocation of GluR1 to these synapses compared with those formed by nonpeptidergic nociceptors. Indeed, neither LTP induced by brief low-frequency C-fiber stimulation in a subset of lamina I projection neurons nor capsaicin-induced potentiation of C-fiber-evoked field potentials in the superficial dorsal horn is fully developed after 30 min, indicating that these forms of potentiation at least partly rely on slow expression mechanisms (Ikeda et al., 2006). However, CaMKII may also potentiate glutamatergic transmission by phosphorylating GluR1 at Ser ${ }^{831}$, which increases the single-channel conductance of homomeric GluR1 receptors (Derkach et al., 2007). Such CaMKII-dependent phosphorylation indeed appears to be elevated in the spinal cord after peripheral capsaicin stimulation (Fang et al., 2002). Therefore, CaMKII-mediated potentiation of synapses formed by $\mathrm{SP}^{+} / \mathrm{CGRP}^{+}$afferent fibers onto lamina I neurons may, at least at an early stage, be mediated by phosphorylation of existing synaptic AMPA receptors rather than accumulation of additional receptors.

In conclusion, we have provided evidence at the ultrastructural level that GluR1-containing AMPA receptors are translocated to the postsynaptic membrane of nonpeptidergic nociceptive primary afferent synapses after acute capsaicin stimulation, suggesting that such synapses are potentiated via an increased density of postsynaptic glutamate receptors in inflammatory hyperalgesia. Furthermore, because CaMKII autophosphorylation within the PSD is concurrently decreased at nonpeptidergic, but 
increased at peptidergic, primary afferent synapses, CaMKII autophosphorylation within this compartment appears to be neither necessary nor sufficient for AMPA receptor incorporation at these synapses.

\section{References}

Braz JM, Nassar MA, Wood JN, Basbaum AI (2005) Parallel "pain” pathways arise from subpopulations of primary afferent nociceptor. Neuron 47:787-793.

Citri A, Malenka RC (2008) Synaptic plasticity: multiple forms, functions, and mechanisms. Neuropsychopharmacology 33:18-41.

Derkach VA, Oh MC, Guire ES, Soderling TR (2007) Regulatory mechanisms of AMPA receptors in synaptic plasticity. Nat Rev Neurosci 8:101-113.

Esteban JA, Shi SH, Wilson C, Nuriya M, Huganir RL, Malinow R (2003) PKA phosphorylation of AMPA receptor subunits controls synaptic trafficking underlying plasticity. Nat Neurosci 6:136-143.

Fang L, Wu J, Lin Q, Willis WD (2002) Calcium-calmodulin-dependent protein kinase II contributes to spinal cord central sensitization. J Neurosci 22:4196-4204.

Galan A, Laird JM, Cervero F (2004) In vivo recruitment by painful stimuli of AMPA receptor subunits to the plasma membrane of spinal cord neurons. Pain 112:315-323.

Guo A, Vulchanova L, Wang J, Li X, Elde R (1999) Immunocytochemical localization of the vanilloid receptor 1 (VR1): relationship to neuropeptides, the $\mathrm{P}_{2} \mathrm{X}_{3}$ purinoceptor and IB4 binding sites. Eur J Neurosci 11:946-958.

Hayashi Y, Shi SH, Esteban JA, Piccini A, Poncer JC, Malinow R (2000) Driving AMPA receptors into synapses by LTP and CaMKII: requirement for GluR1 and PDZ domain interaction. Science 287:2262-2267.

Hunt SP, Mantyh PW (2001) The molecular dynamics of pain control. Nat Rev Neurosci 2:83-91.

Ikeda H, Heinke B, Ruscheweyh R, Sandkühler J (2003) Synaptic plasticity in spinal lamina I projection neurons that mediate hyperalgesia. Science 299:1237-1240.

Ikeda H, Stark J, Fischer H, Wagner M, Drdla R, Jäger T, Sandkühler J (2006) Synaptic amplifier of inflammatory pain in the spinal dorsal horn. Science 312:1659-1662.

Ji RR, Kohno T, Moore KA, Woolf CJ (2003) Central sensitization and LTP: do pain and memory share similar mechanisms? Trends Neurosci 26:696-705.

Julius D, Basbaum AI (2001) Molecular mechanisms of nociception. Nature 413:203-210.

Larsson M, Broman J (2005) Different basal levels of CaMKII phosphorylated at $\mathrm{Thr}^{286 / 287}$ at nociceptive and low-threshold primary afferent synapses. Eur J Neurosci 21:2445-2458.

Larsson M, Broman J (2006) Pathway-specific bidirectional regulation of $\mathrm{Ca}^{2+} /$ calmodulin-dependent protein kinase II at spinal nociceptive synapses after acute noxious stimulation. J Neurosci 26:4198-4205.

Larsson M, Persson S, Ottersen OP, Broman J (2001) Quantitative analysis of immunogold labeling indicates low levels and non-vesicular localization of L-aspartate in rat primary afferent terminals. J Comp Neurol 430:147-159.

Lisman J, Schulman H, Cline H (2002) The molecular basis of CaMKII function in synaptic and behavioural memory. Nat Rev Neurosci 3:175-190.

Liu M, Willmott NJ, Michael GJ, Priestley JV (2004) Differential pH and capsaicin responses of Griffonia simplicifolia IB4 (IB4)-positive and IB4negative small sensory neurons. Neuroscience 127:659-672.

Liu XG, Sandkühler J (1995) Long-term potentiation of C-fiber-evoked potentials in the rat spinal dorsal horn is prevented by spinal $N$-methyl-Daspartic acid receptor blockage. Neurosci Lett 191:43-46.

Mantyh PW, Rogers SD, Honore P, Allen BJ, Ghilardi JR, Li J, Daughters RS, Lappi DA, Wiley RG, Simone DA (1997) Inhibition of hyperalgesia by ablation of lamina I spinal neurons expressing the substance P receptor. Science 278:275-279.

Matsubara A, Laake JH, Davanger S, Usami S, Ottersen OP (1996) Organization of AMPA receptor subunits at a glutamate synapse: a quantitative immunogold analysis of hair cell synapses in the rat organ of Corti. J Neurosci 16:4457-4467.

McLeod AL, Krause JE, Cuello AC, Ribeiro-da-Silva A (1998) Preferential synaptic relationships between substance $\mathrm{P}$-immunoreactive boutons and neurokinin 1 receptor sites in the rat spinal cord. Proc Natl Acad Sci U S A 95:15775-15780.

Merighi A, Polak JM, Theodosis DT (1991) Ultrastructural visualization of glutamate and aspartate immunoreactivities in the rat dorsal horn, with special reference to the co-localization of glutamate, substance $\mathrm{P}$ and calcitonin-gene related peptide. Neuroscience 40:67-80.

Michael GJ, Priestley JV (1999) Differential expression of the mRNA for the vanilloid receptor subtype 1 in cells of the adult rat dorsal root and nodose ganglia and its downregulation by axotomy. J Neurosci 19:1844-1854.

Millan MJ (1999) The induction of pain: an integrative review. Prog Neurobiol 57:1-164.

Nagy GG, Al-Ayyan M, Andrew D, Fukaya M, Watanabe M, Todd AJ (2004) Widespread expression of the AMPA receptor GluR2 subunit at glutamatergic synapses in the rat spinal cord and phosphorylation of GluR1 in response to noxious stimulation revealed with an antigen-unmasking method. J Neurosci 24:5766-5777.

Nichols ML, Allen BJ, Rogers SD, Ghilardi JR, Honore P, Luger NM, Finke MP, Li J, Lappi DA, Simone DA, Mantyh PW (1999) Transmission of chronic nociception by spinal neurons expressing the substance P receptor. Science 286:1558-1561.

Pedersen LM, Lien GF, Bollerud I, Gjerstad J (2005) Induction of long-term potentiation in single nociceptive dorsal horn neurons is blocked by the CaMKII inhibitor AIP. Brain Res 1041:66-71.

Petralia RS, Esteban JA, Wang YX, Partridge JG, Zhao HM, Wenthold RJ, Malinow R (1999) Selective acquisition of AMPA receptors over postnatal development suggests a molecular basis for silent synapses. Nat Neurosci 2:31-36.

Polgár E, Watanabe M, Hartmann B, Grant SG, Todd AJ (2008) Expression of AMPA receptor subunits at synapses in laminae I-III of the rodent spinal dorsal horn. Mol Pain 4:5.

Popratiloff A, Weinberg RJ, Rustioni A (1996) AMPA receptor subunits underlying terminals of fine-caliber primary afferent fibers. J Neurosci 16:3363-3372.

Randić M, Jiang MC, Cerne R (1993) Long-term potentiation and longterm depression of primary afferent neurotransmission in the rat spinal cord. J Neurosci 13:5228-5241.

Ribeiro-da-Silva A (2004) Substantia gelatinosa of the spinal cord. In: The rat nervous system, Ed 3 (Paxinos G, ed). San Diego: Elsevier.

Sandkühler J (2000) Learning and memory in pain pathways. Pain 88:113-118.

Tachibana M, Wenthold RJ, Morioka H, Petralia RS (1994) Light and electron microscopic immunocytochemical localization of AMPA-selective glutamate receptors in the rat spinal cord. J Comp Neurol 344:431-454.

Takumi Y, Ramírez-León V, Laake P, Rinvik E, Ottersen OP (1999) Different modes of expression of AMPA and NMDA receptors in hippocampal synapses. Nat Neurosci 2:618-624.

Todd AJ, Puskar Z, Spike RC, Hughes C, Watt C, Forrest L (2002) Projection neurons in lamina $I$ of rat spinal cord with the neurokinin 1 receptor are selectively innervated by substance $\mathrm{P}$-containing afferents and respond to noxious stimulation. J Neurosci 22:4103-4113.

Tominaga M, Caterina MJ, Malmberg AB, Rosen TA, Gilbert H, Skinner K, Raumann BE, Basbaum AI, Julius D (1998) The cloned capsaicin receptor integrates multiple pain-producing stimuli. Neuron 21:531-543.

Vierck CJ Jr, Kline RH, Wiley RG (2003) Intrathecal substance P-saporin attenuates operant escape from nociceptive thermal stimuli. Neuroscience 119:223-232.

Willis WD, Coggeshall RE (2004) Sensory mechanisms of the spinal cord, Ed 3. New York: Kluwer Academic/Plenum.

Yang HW, Hu XD, Zhang HM, Xin WJ, Li MT, Zhang T, Zhou LJ, Liu XG (2004) Roles of CaMKII, PKA, and PKC in the induction and maintenance of LTP of C-fiber-evoked field potentials in rat spinal dorsal horn. J Neurophysiol 91:1122-1133.

Zeitz KP, Giese KP, Silva AJ, Basbaum AI (2004) The contribution of autophosphorylated alpha-calcium-calmodulin kinase II to injury-induced persistent pain. Neuroscience 128:889-898.

Zhang X, Nicholas AP, Hökfelt T (1993) Ultrastructural studies on peptides in the dorsal horn of the spinal cord-I. Co-existence of galanin with other peptides in primary afferents in normal rats. Neuroscience 57:365-384.

Zhu JJ, Esteban JA, Hayashi Y, Malinow R (2000) Postnatal synaptic potentiation: delivery of GluR4-containing AMPA receptors by spontaneous activity. Nat Neurosci 3:1098-1106. 